\title{
Energy Dependent Growth of Nucleon and Inclusive Charged Hadron Distributions*
}

\author{
WANG Hong-Min ${ }^{1 \dagger} \dagger$, HOU Zhao-Yu², SUN Xian-Jing ${ }^{3}$ \\ ${ }^{1}$ Physics Department, Academy of Armored Forces Engineering of PLA, Beijing 100072, China \\ ${ }^{2}$ Physics Graduate School, Shijiazhuang Railway Institute, 050043, China \\ ${ }^{3}$ Institute of High Energy Physics, Chinese Academy of Sciences, Beijing 100049, China
}

\begin{abstract}
In the Color Glass Condensate formalism, charged hadron $p_{\mathrm{T}}$ distributions in $\mathrm{p}+\mathrm{p}$ collisions are studied by considering an energy-dependent broadening of nucleon's density distribution. Then, in the Glasma flux tube picture, the $n$-particle multiplicity distributions at different pseudo-rapidity ranges are investigated. Both of the theoretical results show good agreement with the recent experimental data from ALICE and CMS at $\sqrt{s}=0.9,2.36,7 \mathrm{TeV}$. The predictive results for $p_{\mathrm{T}}$ and multiplicity distributions in $\mathrm{p}+\mathrm{p}$ and $\mathrm{p}+\mathrm{Pb}$ collisions at the Large Hadron Collider are also given in this paper.
\end{abstract}

Key words: charged hadron $p_{\mathrm{T}}$ distributions, Glasma flux tube, gluon saturation model

PACS numbers: 25.75.-q, 24.85.+p, 14.20.Dh

\section{Introduction}

The measurement of charged hadron transverse momentum $\left(p_{\mathrm{T}}\right)$ spectrum in heavy-ion collisions is a very significant topic on experimental physics. Recently, the ALICE and CMS experiments at the Large Hadron Collider (LHC) have given the data for charged hadron $p_{\mathrm{T}}$ distributions in $\mathrm{p}+\mathrm{p}$ collisions at $\sqrt{s}=0.9,2.36,7 \mathrm{TeV}[1-2]$. The experimental results show that the scaling features of charged hadrons previously observed at lower energies do not hold good at high energies. These indicate that we still can not give some basic understanding of the mechanism involved in the charged particle production. Thus, a systematic theoretical study on this topic is still very necessary.

An effective theory to study charged hadron $p_{\mathrm{T}}$ spectrum is the Color Glass Condensate (CGC) approach [3]. The CGC is a state predicted by quantum chromodynamics (QCD) at high energies where gluons in a hadron wave function expect to statute. The cornerstone of the CGC approach is the existence of a hadron saturation scale $Q_{s}$ at which gluon recombination effects start to balance gluon radiation. In order to give an accurate theoretical analysis, the nucleon's density distribution in position space must be considered. Thus, the saturation scale, $Q_{s}$, should be considered as a function of the nucleon's thickness function, $T_{\mathrm{p}}(b)$. Furthermore, due to gluon saturation, the width of the gluon distribution inside a nucleon should grow with collision energy, $\sqrt{s}$ [4]. This will lead to

\footnotetext{
* Supported by Natural Science Foundation of Hebei Province (A2012210043)

†E-mail: whmw@sina.com.cn
} 
a broadening of the nucleon's density distribution in position space as $\sqrt{s}$ increases. Therefore, an energy-dependent broadening of the nucleon's thickness function in the $Q_{s}$ should also be considered.

Based on the CGC theory, many phenomenological saturation models, such as the Golec-Biernat and Wüsthoff (GBW) model [5], the Kharzeev, Levin and Nardi (KLN) model [6], the CGC model [7] and the Kovchegov, Lu and Rezaeian (KLR) model [8], are established. It should be noted that the KLR model is based on the Anti-de Sitter space/conformal field theory (AdS/CFT). In this paper, the KLN and KLR-AdS/CFT model are used because the simple analytic unintegrated gluon distribution functions can be obtained by a Fourier transform from the dipole amplitude of these two models.

Integrating charged hadron transverse momentum distributions over $\mathbf{p}_{\mathrm{T}}$, we can obtain the impact parameter (b) dependent mean multiplicity, $\bar{n}(\mathbf{b})$. The mean multiplicity and the parameter controlling the size of the fluctuations, $k$, are two parameters that characterize negative binominal distribution (NBD) [9-10]. In the CGC picture, particles produced locally in the transverse plane are considered as correlating by approximately boost invariant flux tubes [11]. Since there are $Q_{S}^{2} S_{\perp}$ such flux tubes emitting gluons, the parameter $k$ should be proportional to $Q_{S}^{2} S_{\perp}$, where $S_{\perp}$ is the transverse overlap area of two collision hadrons. This indicates that the parameter $k$ in the NBD is also a function of the thickness function, $T_{\mathrm{p}}(b)$, and influenced by the energy-dependent broadening. By convolving the NBD and the probability for an inelastic collision over the impact parameter, the $n$-particle multiplicity distributions can be obtained.

\section{Energy Dependent Growth of Nucleon and Charged Hadron $p_{\mathrm{T}}$ Distributions}

In hadron-hadron collisions, the transverse momentum distributions for charged hadrons at leading order can be expressed as [12]

$$
\begin{aligned}
\frac{d N(\mathbf{b})}{d^{2} \mathbf{p}_{T}}=\frac{1}{C_{F}} \frac{1}{p_{T}^{2}} \int & d y \int d^{2} \mathbf{r}_{T} \int_{0}^{p_{T}} d^{2} \mathbf{k}_{T} \alpha_{s}\left(Q^{2}\right) \phi_{1}\left(x_{1}, \frac{\left(\mathbf{k}_{T}+\mathbf{p}_{T}\right)^{2}}{4}, \mathbf{b}\right) \\
& \times \phi_{2}\left(x_{2}, \frac{\left(\mathbf{k}_{T}-\mathbf{p}_{T}\right)^{2}}{4}, \mathbf{b}-\mathbf{r}_{\perp}\right),
\end{aligned}
$$

where $N_{c}=3, x_{1,2}=\left(p_{T} / \sqrt{s}\right) \exp ( \pm y)$, and $C_{F}=\left(N_{c}^{2}-1\right) /\left(2 \pi^{3} N_{c}\right)$. The running coupling constant $\alpha_{s}\left(Q^{2}\right)=\min \left\{\frac{4 \pi}{9 \ln \left[Q^{2} / \Lambda_{\mathrm{QCD}}^{2}\right]}, 0.5\right\}$ with $\Lambda_{\mathrm{QCD}}=0.2 \mathrm{GeV}$ and $Q^{2}=\max \left\{\left(\mathbf{k}_{T}+\mathbf{p}_{T}\right)^{2}\right) / 4,\left(\mathbf{k}_{T}-\right.$ $\left.\left.\left.\mathbf{p}_{T}\right)^{2}\right) / 4\right\}$. $\mathbf{b}$ and $\mathbf{r}_{\perp}$ are the impact factor and the transverse position of the gluon, respectively. as $[6]$

In Eq.(1), $\phi$ is the unintegrated gluon distribution (UGD). In the KLN model, $\phi$ can be taken

$$
\phi\left(x, k^{2}, \mathbf{b}\right)=\frac{\xi C_{F} Q_{s}^{2}}{\alpha_{s}\left(Q_{s}^{2}\right)}\left\{\begin{array}{ll}
\frac{1}{Q_{s}^{2}+\Lambda^{2}}, & k \leq Q_{s} \\
\frac{1}{k^{2}+\Lambda^{2}}, & k>Q_{s}
\end{array},\right.
$$

where $\xi$ is a normalization factor, and the saturation scale [4-5]

$$
Q_{s}^{2}(x)=Q_{0}^{2}\left(\frac{0.01}{x}\right)^{\lambda}
$$

where $Q_{0}^{2}=2 \mathrm{GeV}^{2}, \lambda=0.288$. In the KLR-AdS/CFT model, $\phi$ can be obtained by a Fourier transform from the dipole-nucleus amplitude given in Ref.[8]

$$
\phi^{\operatorname{AdS}}\left(x, k^{2}, \mathbf{b}\right)=\int d^{2} \mathbf{r} e^{i \mathbf{k} \cdot \mathbf{r}} N(x, r)=\frac{32 \pi}{\left(Q_{s}^{\mathrm{AdS}}\right)^{2}} \frac{1}{\left[1+16 k^{2} /\left(Q_{s}^{\mathrm{AdS}}\right)^{2}\right]^{3 / 2}},
$$


where the corresponding saturation scale

$$
Q_{s}^{\mathrm{AdS}}(x)=\frac{2 A_{0} x}{M_{0}^{2}(1-x) \pi}\left(\frac{1}{\rho_{m}^{3}}+\frac{2}{\rho_{m}}-2 M_{0} \sqrt{\frac{1-x}{x}}\right) .
$$

The parameter $\rho_{m}, M_{0}$ and $A_{0}$ in Eq.(5), as given in Ref.[8], can be obtained by a fit to HERA data.

In order to give an accurate theoretical analysis, the UGD should be considered as a function of the transverse position distribution of the nucleon. Thus, the gluon saturation momentum is always written as [4]

$$
Q_{s, \mathrm{p}(\mathrm{A})}^{2}(x, \mathbf{b})=Q_{s}^{2}(x)\left(\frac{T_{\mathrm{p}(\mathrm{A})}(\mathbf{b})}{T_{\mathrm{p}(\mathrm{A}), 0}}\right),
$$

where the nuclear thickness function

$$
T_{\mathrm{p}(\mathrm{A})}(\mathbf{b})=\int d z \rho_{\mathrm{p}(\mathrm{A})}(\mathbf{b}, z),
$$

and $T_{\mathrm{p}(\mathrm{A}), 0}=1.53 \mathrm{fm}^{-2}$ [4]. For the nuclear density distribution, we use the Woods-Saxon form [13]

$$
\rho_{\mathrm{A}}(\mathbf{b}, z)=\frac{\rho_{0}}{1+\exp [(r-R) / a]},
$$

where $r=\sqrt{\mathbf{b}^{2}+z^{2}}$, and the measured values for $\mathrm{Pb}$ are $\rho_{0}=0.1612, R=6.62 \mathrm{fm}, a=0.545 \mathrm{fm}$. For the proton's density distribution, the Gaussian form is used

$$
\rho_{\mathrm{p}}(\mathbf{b}, z)=\frac{e^{-r^{2} /(2 B)}}{(2 \pi B)^{3 / 2}}
$$

Because of gluon saturation, the inelastic nucleon-nucleon cross section $\sigma_{\text {in }}$ should grow as $\sqrt{s}$ increases. This will result in a broadening of the nucleon's density distribution in position space. Therefore, the Gaussian width $B$ should be written as a function of $\sqrt{s}$ [4]

$$
B(\sqrt{s})=\frac{\sigma_{\text {in }}(\sqrt{s})}{14.30} \mathrm{fm}^{2},
$$

with $\sigma_{\text {in }}(\sqrt{s})=52,60,70.45,72,76.3 \mathrm{mb}$ at $\sqrt{s}=0.9,2.36,7,8.8,14 \mathrm{TeV}$, respectively [9,14-15].

In this paper, we also assume the gluon saturation scale should have a small dependence on $\sqrt{s}$ through the 3 -dimensional rms radius of the proton [16]

$$
Q_{s, \mathrm{p}(\mathrm{A})}^{2}(\sqrt{s})=Q_{s, \mathrm{p}(\mathrm{A})}^{2}\left(\sqrt{s_{0}}\right)\left(\frac{\pi r_{\mathrm{rms}, 0}^{2}}{\pi r_{\mathrm{rms}}^{2}}\right)^{1 / \delta}
$$

where $\delta=0.8$ and the 3 -dimensional rms radius $r_{\mathrm{rms}}=\sqrt{<r^{2}>}=\sqrt{3 B}$.

\section{Negative Binomial Distribution in the Glasma Flux Tube Picture}

Negative binomial multiplicity distribution is an interesting and important feature observed in multiplicity distribution of charged hadron production in high energy collisions. In the Glasma flux tube framework [11], the negative binomial distribution can be derived as

$$
P_{n}^{\mathrm{NBD}}(\bar{n}, k)=\frac{\Gamma(k+n)}{\Gamma(k) \Gamma(n+1)}\left(\frac{\bar{n}}{k}\right)^{n}\left(1+\frac{\bar{n}}{k}\right)^{-n-k},
$$


where the parameter $k$ and mean multiplicity $\bar{n}$ are all considered as functions of the impact parameter. The parameter $k$ in the saturation approach is defined to be

$$
k(\mathbf{b})=\zeta \frac{\left(N_{c}^{2}-1\right) Q_{s, \mathrm{p}}^{2} S_{\perp}(\mathbf{b})}{2 \pi},
$$

where $\zeta$ is a dimensionless parameter and $S_{\perp}$ is the overlap area of the two hadrons [11]. For a given impact parameter $\mathbf{b}$,

$$
Q_{s, \mathrm{p}}^{2} S_{\perp}(\mathbf{b})=\int \mathrm{d}^{2} \mathbf{s}_{\perp} Q_{s, \mathrm{p}}^{2}\left(\mathbf{s}_{\perp}, \mathbf{b}\right),
$$

where $Q_{s, \mathrm{p}}$ in the overlap area of the two hadrons is chosen to be $Q_{s, \mathrm{p}}\left(\mathbf{s}_{\perp}, \mathbf{b}\right)=\min \left\{Q_{s, \mathrm{p}}\left(\mathbf{s}_{\perp}\right), Q_{s, \mathrm{p}}\left(\mathbf{s}_{\perp}-\right.\right.$ b) $\}$. The mean multiplicity $\bar{n}$ can be obtained by integrating Eq.(1) over $\mathbf{p}_{T}$

$$
\bar{n}(\mathbf{b})=C_{\mathrm{m}} \int d^{2} \mathbf{p}_{T} \frac{d N(\mathbf{b})}{d^{2} \mathbf{p}_{T}}
$$

where the pre-factor $C_{\mathrm{m}}$ is proportional to $\sigma_{\mathrm{in}}$.

In order to compute the probability distribution as a function of multiplicity, one should convolve the NBD at a given impact parameter (Eq.(11)) with the probability for an inelastic collision $\left(\frac{d P_{\text {inel }}}{d^{2} \mathbf{b}}\right)$ at the same impact parameter. Thus, the probability distribution for producing $n$ particle can be given by

$$
P(n)=\int d^{2} \mathbf{b} \frac{d P_{\text {inel }}}{d^{2} \mathbf{b}} P_{n}^{\mathrm{NBD}}(\bar{n}(\mathbf{b}), k(\mathbf{b})) .
$$

where the probability distribution can be given in impact parameter eikonal models [17]

$$
\frac{d P_{\text {inel }}}{d^{2} \mathbf{b}}=\frac{1-\exp \left(-\sigma_{\mathrm{gg}} T_{\mathrm{pp}}\right)}{\int d^{2} \mathbf{b}\left[1-\exp \left(-\sigma_{\mathrm{gg}} T_{\mathrm{pp}}\right)\right]}
$$

with the energy dependent quantity $\sigma_{\mathrm{gg}}=4 \pi \lambda B$ [4]. The overlap function for two protons at a given impact parameter can be expressed as

$$
T_{\mathrm{pp}}(\mathbf{b})=\int d^{2} \mathbf{s}_{\perp} T_{\mathrm{p}}\left(\mathbf{s}_{\perp}\right) T_{\mathrm{p}}\left(\mathbf{s}_{\perp}-\mathbf{b}\right) .
$$

\section{Results and Discussion}

Charged hadron transverse momentum distributions in $\mathrm{p}+\mathrm{p}$ collisions at different collision energies are shown in Fig.1. Fig.1 (a) and (b) are the results with the KLN and the KLR-AdS/CFT model, respectively. The $p_{\mathrm{T}}$ distributions are averaged over the $\eta$ range from -2.4 to 2.4. The rapidity $(y)$ in Eq.(1) can be changed into the pseudo-rapidity $(\eta)$ using the transformation

$$
y(\eta)=\frac{1}{2} \ln \frac{\sqrt{\cosh ^{2} \eta+m_{0}^{2} / p_{T}^{2}}+\sinh \eta}{\sqrt{\cosh ^{2} \eta+m_{0}^{2} / p_{T}^{2}}-\sinh \eta},
$$

where $m_{0}\left(\sim \Lambda_{\mathrm{QCD}}\right)$ is the rest mass of particle. The experimental data come from CMS and ALICE [1-2]. The parameter $\xi(=0.51)$ in Eq. (2) can be obtained by a $\chi^{2}-$ analysis with the experimental data [18-19]. In Fig.1 (a), it is shown that the theoretical results with the KLN model are in good agreement with the experimental data. For the KLR-AdS/CFT model, as shown in Fig.1 (b), the 
theoretical results fit to the experimental only at $\sqrt{s}=7 \mathrm{TeV}$ while $p_{\mathrm{T}}$ is small. The reason is that the KLR-AdS/CFT model is an effective model at small Bjorken- $x\left(x<10^{-4}\right)$. At large $p_{\mathrm{T}}$ or small $\sqrt{s}, x_{1,2}\left(=p_{\mathrm{T}} / \sqrt{s} \cdot \exp ( \pm y)\right)$ is out of the valid range of this model. In Fig.2, the predictive results with the KLN model for charged hadron $p_{\mathrm{T}}$ distributions in $\mathrm{p}+\mathrm{Pb}$ (solid curves) and $\mathrm{p}+\mathrm{p}$ (dashed curves) collisions are also given. Fig.2 (a) and (b) are the results at $\sqrt{s}=5.02 \mathrm{TeV}$ and $8.8 \mathrm{TeV}$, respectively. It is shown that the results for $\mathrm{p}+\mathrm{Pb}$ collisions are closer to the values of $\mathrm{p}+\mathrm{p}$ collisions at small $p_{\mathrm{T}}$ than those at large $p_{\mathrm{T}}$.

Fig.3 (a) shows the the probability distribution for an inelastic collision at $b$. The curves are the results at $\sqrt{s}=0.9 \mathrm{TeV}$ (solid curve), $2.36 \mathrm{TeV}$ (dashed curve), $7 \mathrm{TeV}$ (dash-dotted curve), and 14 $\mathrm{TeV}$ (dotted curve). The results show that the probability distributions $2 \pi b d P_{\text {inel }} / d^{2} \mathbf{b}_{\perp}$ at certain $\sqrt{s}$ have a sharply peaked distribution and the impact parameter $b$ of the peak grows with increasing collision energy $\sqrt{s}$. Fig.3 (b) shows the width parameter $k$ versus impact parameter $b$. The figure captions are the same as those in Fig.3 (a). It is shown that the width parameter $k$ becomes larger as $\sqrt{s}$ increases. This is because the number of flux tubes $Q_{S}^{2} S_{\perp}$ in $k$ depends on the energy-dependent broadening thickness function $T_{\mathrm{p}}$.

The results for charged hadron multiplicity distributions in the range of $|\eta|<0.5$ and $|\eta|<1$ are shown in Fig.4. The figures are the results at $\sqrt{s}=0.9 \mathrm{TeV}$ (a), $2.36 \mathrm{TeV}$ (b), $7 \mathrm{TeV}$ (c), and $14 \mathrm{TeV}$ (d). The solid curves are the results with the Glasma flux tube approach, and the dashed curves are the results without considering the nucleon's transverse position distribution. The data come from ALICE [20]. The parameter $\zeta=0.05$ is extracted from a fit to data at $\sqrt{s}=2.36 \mathrm{TeV}$ and used for all other energies. It is shown that the theoretical results with the Glasma flux tube approach are in good agreement with the experimental data at all collision energies. For the method without considering the transverse distribution, the theoretical results fit well to the data at $\sqrt{s}=$ $2.36 \mathrm{TeV}$ but show deviations for other collisions at highest multiplicities.

In summary, charged hadron $p_{\mathrm{T}}$ distributions in $\mathrm{p}+\mathrm{p}$ collisions at various collision energies are studied with the CGC approach. By considering an energy-dependent broadening of the nucleon's density distribution in position space, the theoretical results of the KLN model are in good agreement with the experimental data from CMS and ALICE. Then, the probability distribution for producing $n$ particle at different pseudo-rapidity ranges are also studied in the picture of Glasma flux tube, and the results fit well to the data. The predictive results for $p_{\mathrm{T}}$ and multiplicity distributions in $\mathrm{p}+\mathrm{p}$ and $\mathrm{p}+\mathrm{Pb}$ collisions will be examined by the forthcoming experiment at the LHC. 


\section{References}

[1] CMS Collaboration. Phys. Rev. Lett., 2010, 105: 022002

[2] ALICE Collaboration. Phys. Lett. B, 2010, 688: 21

[3] Gelis F, Iancu E, Jalilian-Marian J et al. Ann. Rev. Nucl. Part. Sci., 2010, 60: 463

[4] Heinz U, Moreland J S. Phys. Rev. C, 2011, 84: 054905

[5] Golec-Biernat K, Wüsthoff M. Phys. Rev. D, 1999, 59: 014017

[6] Kharzeev D, Levin E, Nardi M. Nucl. Phys. A, 2004, 730: 448

[7] Iancu E, Itakura K, Munier S. Phys. Lett. B, 2004, 590: 199

[8] Kovchegov Y V, LU Z, Rezaeian A H. Phys. Rev. D, 2009, 80: 074023

[9] Dumitru A, Kharzeev D E, Levin E M et al. Phys. Rev. C, 2012, 85: 044920

[10] Premomoy G. Phys. Rev. D, 2012, 85: 054017

[11] Tribedy P, Venugopalan R. Nucl. Phys. A, 2011, 850: 136

[12] Kuhlman A, Heinz U, Kovchegov Y V. Phys. Lett. B, 2006, 638: 171

[13] deVries H, deJager C W, deVries C. At. Data Nucl. Data Tables, 1987, 36: 495; Miller M L, Reygers K, Sanders S J et al. Ann. Rev. Nucl. Part. Sci., 2007, 57: 205

[14] Albacete J L, Dumitru A, Fujii H et al. Nucl. Phys. A, 2013, 897: 1

[15] Block M M, Halzen F. Phys. Rev. D, 2011, 83: 077901

[16] Armesto N, Salgado C A, Wiedemann U A. Phys. Rev. Lett., 2005, 94: 022002

[17] d'Enterria D, Eyyubova G K, Korotkikh V L et al. Eur. Phys. J. C, 2010, 66: 173

[18] WANG H M, HOU Z Y, ZHANG B A et al. Int. J. Mod. Phys. A, 2010, 25: 1009

[19] WANG H M, HOU Z Y , SUN X J. Nucl. Sci. Tech., 2014, 25: 040502

[20] Aamodt K, Abel N, Abeysekara U et al. Eur. Phys. J. C, 2010, 68: 345 


\section{Figure Captions}

Figure1: Charged hadron $p_{\mathrm{T}}$ distributions for $\mathrm{p}+\mathrm{p}$ collisions averaged over the range of $|\eta|<2.4$ with the KLN model (a) and the KLR-AdS/CFT model (b). The experimental data come from CMS and ALICE [1-2].

Figure2: The predictive results for charged hadron $p_{\mathrm{T}}$ distributions at $\sqrt{s}=5.02 \mathrm{TeV}$ (a) and 8.8 TeV (b). The sold and dashed curves are the results for $\mathrm{p}+\mathrm{Pb}$ and $\mathrm{p}+\mathrm{p}$ collisions, respectively.

Figure3: The probability distribution for inelastic collision (a) and the width parameter $k$ (b) as a function of impact parameter $b$.

Figure4: Multiplicity distributions of charged hadrons in the range of $|\eta|<0.5$ and $|\eta|<1$. The figures are the results at $\sqrt{s}=0.9 \mathrm{TeV}$ (a), $2.36 \mathrm{TeV}$ (b), $7 \mathrm{TeV}$ (c) and $14 \mathrm{TeV}$ (d). The solid curves are the results with the Glasma flux tube approach, and the dashed curves are the results without considering nucleon's position distribution. The data come from ALICE [20]. 

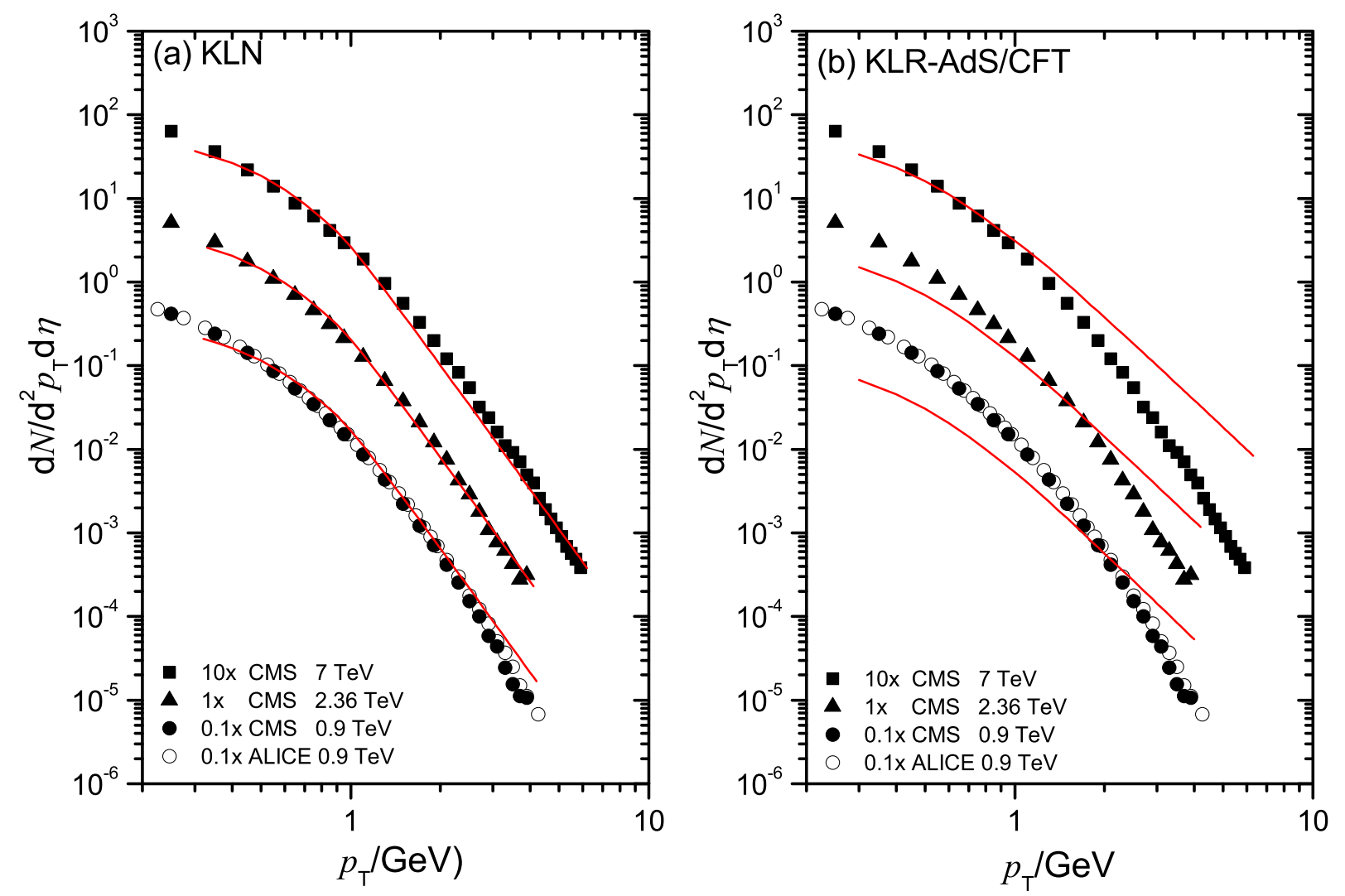

Figure 1 

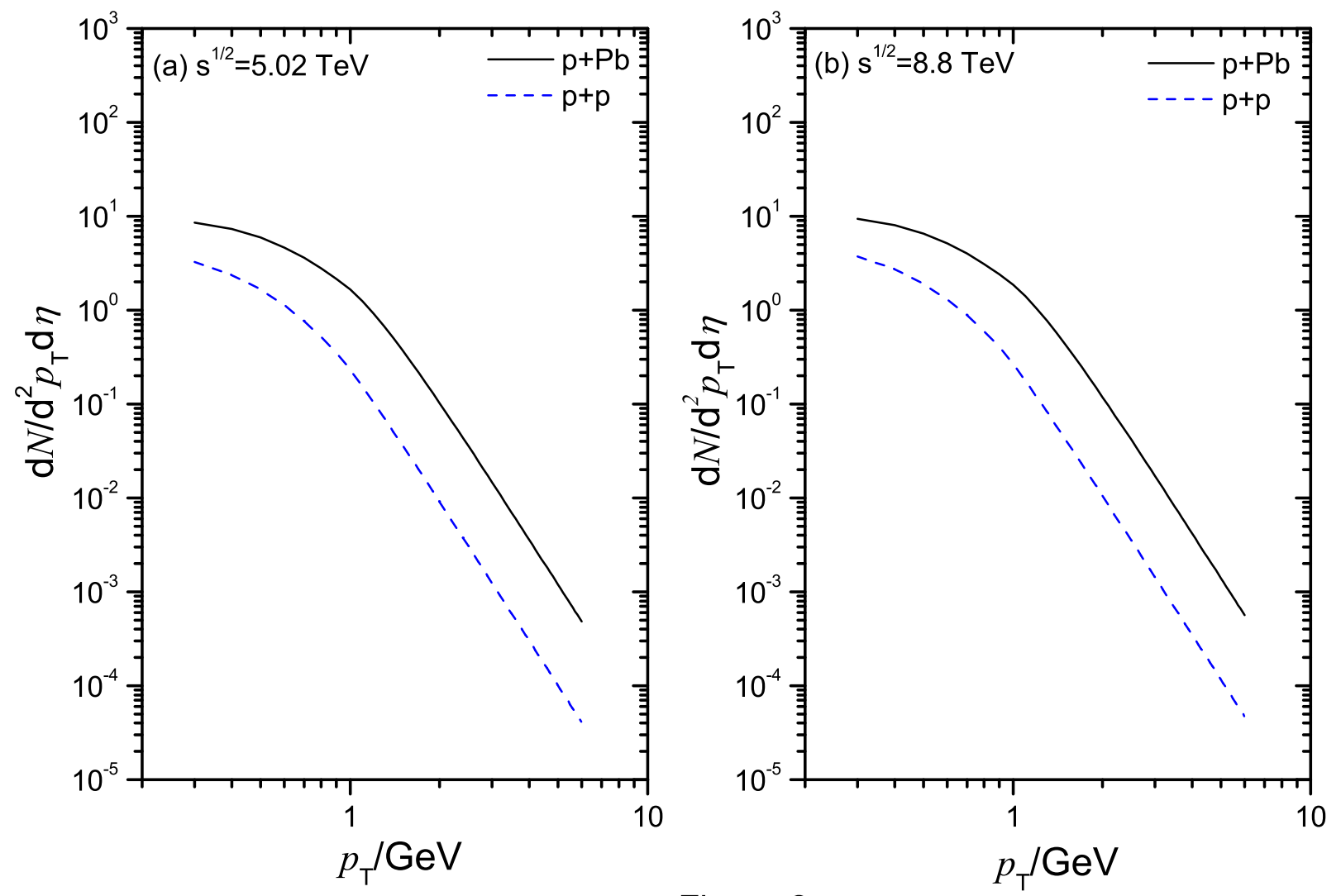

Figure 2 

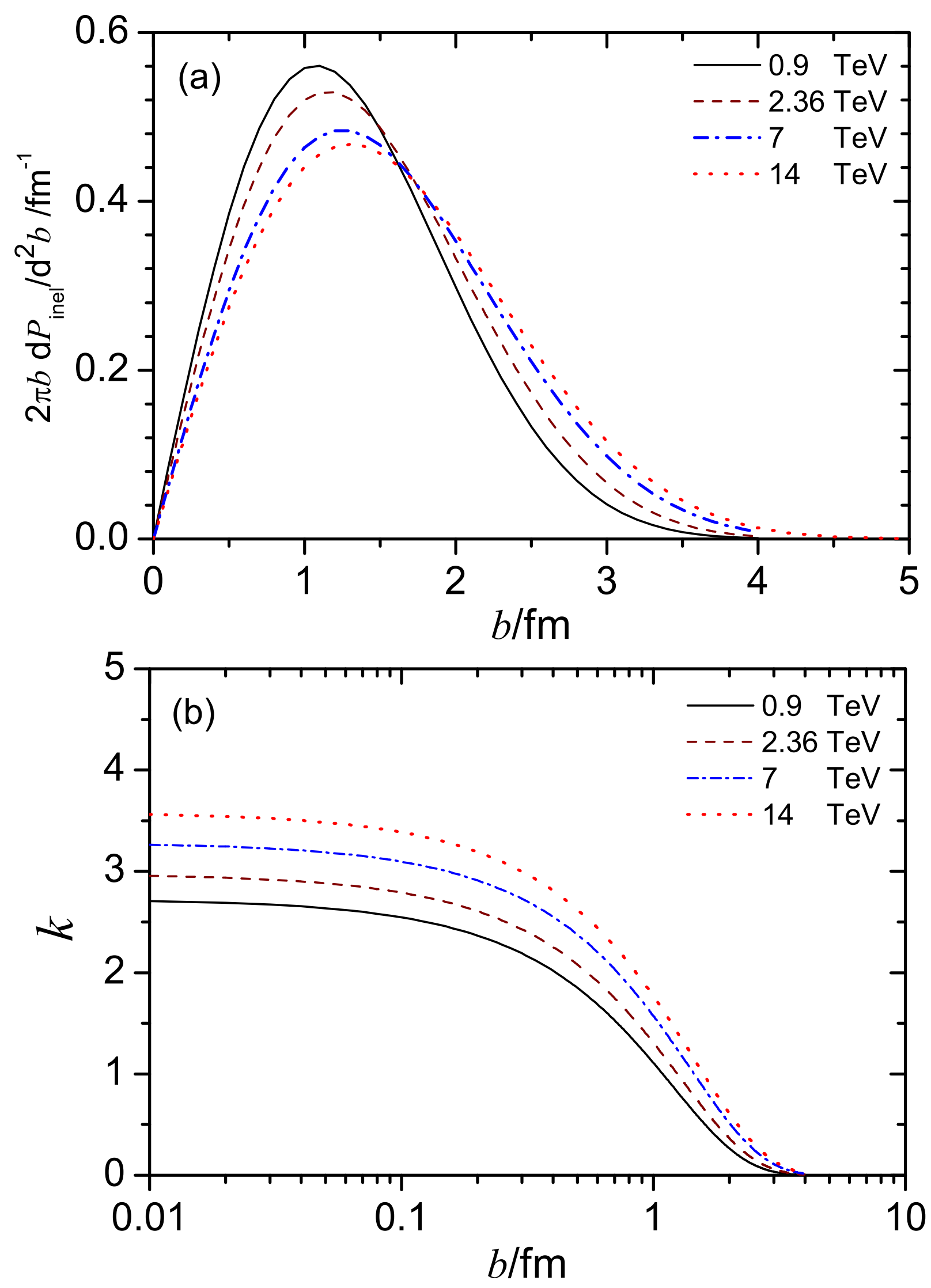

Figure 3 

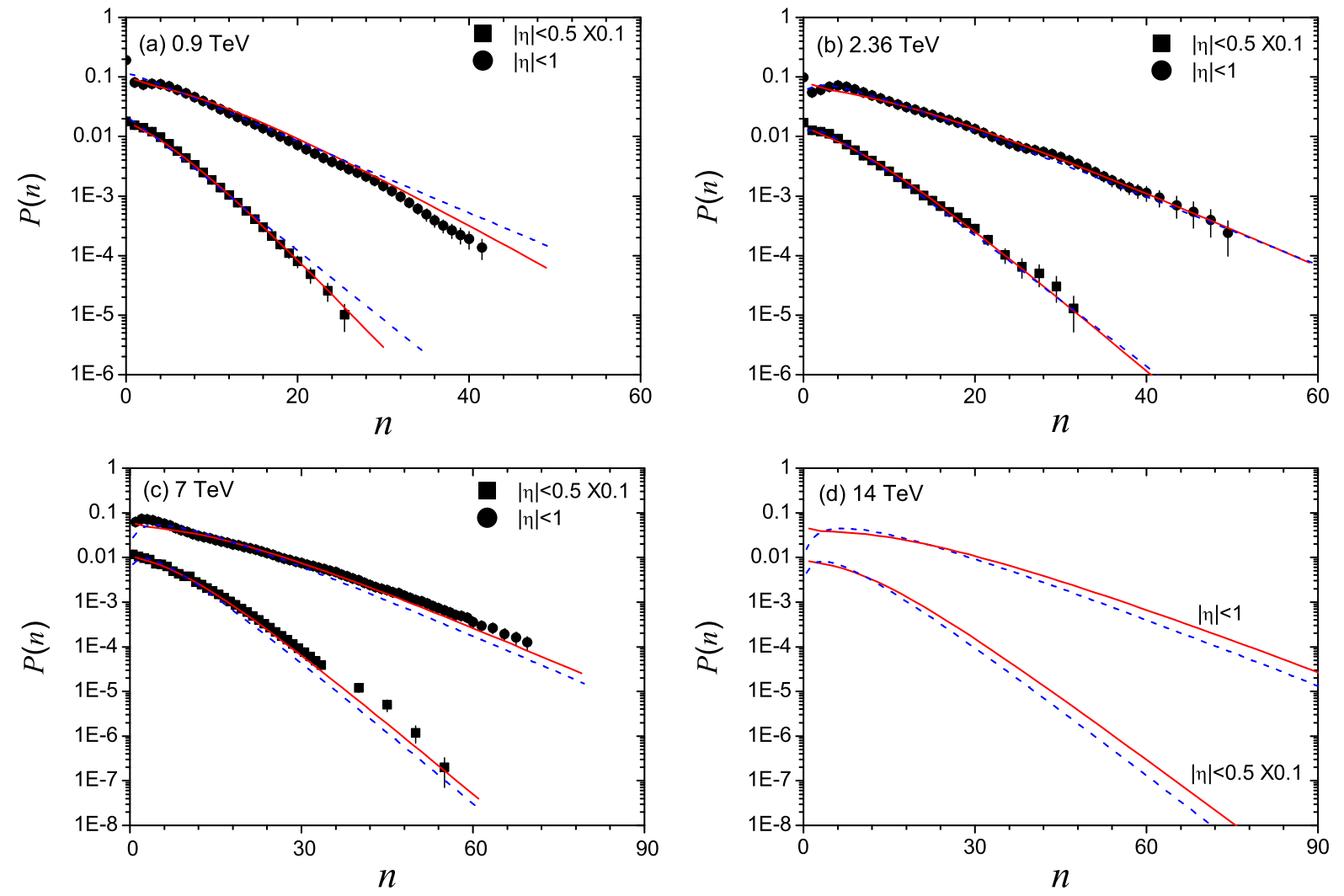

Figure 4 